CARNETS OE Carnets de géographes

GÉOGRAPHES: $\quad 10 \mid 2017$

Pour une réflexion collective sur l'enseignement de la géographie à l'Université

\title{
Adolescences "Em Sampa"
}

Représentations urbaines de lycéens français expatriés à São Paulo

Nolwenn Azilis Rigollet

\section{OpenEdition}

Journals

Édition électronique

URL : http://journals.openedition.org/cdg/1214

DOI : $10.4000 /$ cdg. 1214

ISSN : 2107-7266

Éditeur

UMR 245 - CESSMA

Référence électronique

Nolwenn Azilis Rigollet, « Adolescences "Em Sampa" », Carnets de géographes [En ligne], 10 | 2017, mis en ligne le 30 septembre 2017, consulté le 20 avril 2019. URL : http://journals.openedition.org/ cdg/1214; DOI : 10.4000/cdg.1214

Ce document a été généré automatiquement le 20 avril 2019

La revue Carnets de géographes est mise à disposition selon les termes de la Licence Creative Commons Attribution - Pas d'Utilisation Commerciale - Pas de Modification 4.0 International. 


\title{
Adolescences "Em Sampa"
}

\author{
Représentations urbaines de lycéens français expatriés à São Paulo
}

\author{
Nolwenn Azilis Rigollet
}

\section{Introduction}

1 Les migrations internationales sont au centre de travaux de géographes depuis bon nombre d'années. Pourtant, ces travaux sur les mobilités intègrent peu la question des enfants et des adolescents puisque ces derniers « suivent » leurs parents, ceux qui sont au cœur des réflexions sur les mobilités. Le présupposé sous-jacent est que ces mobilités enfantines et adolescentes relèvent de la sphère privée, et donc ne concernent pas les espaces productifs visibles. Issu d'un travail de terrain de Master II (Pays émergents et en Développement ${ }^{1}$ )- cet article vise à mettre en lumière une catégorie de jeunes comme sujet de recherche en géographie. A ce titre je me suis attachée à tenter de comprendre les représentations de l'espace paulistain pour des lycéens français expatriés.

\section{Le terrain paulistain}

2 Enseignante dans le secondaire dans un lycée du Val-de-Marne, je travaille au quotidien avec des adolescents de 14 ans à 18 ans. Leurs représentations du Monde m'interpellent. De retour d'un premier séjour d'études au Brésil en 2013, j'ai été particulièrement sensible à la perception que mes élèves avaient de ce pays et de ses villes en particulier. En tant que métropole émergente, la ville brésilienne représente en effet pour les élèves un cliché des métropoles des Suds: une cité dangereuse sans planification urbaine. En quête d'un terrain pour mon travail de recherche de master, il m'a semblé stimulant de pouvoir revenir à São Paulo et de chercher à savoir si des adolescents français vivant l'expérience de l'expatriation avaient eux aussi cette perception qui serait à la fois influencée par des recommandations exogènes (recommandations des ambassades et des entreprises), familiales (poids de la parentalité dans les mobilités des adolescents) et par leur expérience du quotidien dans la ville. L'opportunité d'un stage à l'été 2014 au Lycée Pasteur- le lycée franco-brésilien de São Paulo- m'a permis de mener un travail de terrain 
avec des lycéens y étant scolarisés. L'établissement accueille un tiers de binationaux Français-Brésiliens, un tiers de Français et un tiers de Brésiliens ou autres nationalités. Pour cette étude, des élèves de deux classes de seconde âgés de 13 à 15 ans, soit 47 élèves ont participé à un travail de cartes mentales. Parmi eux, 47 \% étaient de sexe masculin et $53 \%$ de sexe féminin. J'ai aussi mené des entretiens qualitatifs d'une moyenne de 45 minutes avec 40 élèves français sur le total des 184 élèves scolarisés dans l'établissement soit $21,7 \%$ du total des élèves et les deux-tiers des lycéens expatriés. Les entretiens se sont réalisés au CDI du lycée, sur la base du volontariat. 70\% des interviewés sont des filles alors qu'elles représentaient $52 \%$ des effectifs de seconde, première et terminale du Lycée Pasteur lors de l'année scolaire 2014. La durée de la présence de ces élèves à São Paulo s'étale de 6 mois à 7 ans.

3 Le référent spatial que constitue l'État-nation se trouve aujourd'hui confronté à l'intensification des interactions des sociétés à l'échelle mondiale. L'accroissement des mobilités internationales contribue à redéfinir les rapports qui unissent les populations à leur territoire d'appartenance (Lévy, 2008), ce que vient confirmer la très forte augmentation du nombre de Français expatriés. Au 31 décembre 2014, on comptait 1680 594 inscrits au Registre des Français de l'étranger (1 642953 au 31 décembre 2013). Au Brésil 21509 Français avaient été enregistrés pour l'année 2014 soit une augmentation de 3\% par rapport aux 20806 de l'année précédente ${ }^{2}$. La communauté française au Brésil est estimée à 30000 personnes. Au moment du terrain, près de la moitié réside dans l'État de São Paulo (10 566 inscrits), on compte 7340 inscrits à Rio de Janeiro, tandis que c'est dans la circonscription de Brasília (la plus vaste) que les Français sont les moins nombreux (3 603 personnes). Plus de 350 filiales d'entreprises françaises sont implantées au Brésil (dont $80 \%$ dans la circonscription de São Paulo). La plupart des grands groupes français sont représentés. Les parents (le père majoritairement) de la majeure partie des élèves rencontrés travaillent pour un grand groupe français. Il est à noter que les élèves interrogés ne sont dans la ville que pour une courte durée (la durée moyenne des contrats d'expatriation étant de trois années).

\section{São Paulo : « métropole corporative fragmentée » (Santos, 1990)}

Dans les années 1940, 1,3 million d'habitants étaient dénombrés à São Paulo alors qu'au début du 21ème siècle la population atteint 10,4 millions (soit une augmentation de 700\%) en six décennies. Maria Adélia Aparecida de Souza a noté dans ses travaux que depuis l'industrialisation, le développement paulistain a pris la forme de deux processus parallèles : un processus de ségrégation de l'espace qui intéresse les classes sociales les plus aisées et les classes moyennes, ce que De Souza appelle «la ville du capital », et la "ville de la révolution ", c'est à dire l'espace des pauvres (De Souza, 1998). Au cours des dernières années, le développement urbain de São-Paulo a été bouleversé par une tertiarisation de l'économie. La saturation de l'axe central des affaires à São Paulo a provoqué un déplacement vers d'autres quartiers et une spéculation immobilière et foncière. Avec un coefficient de Gini supérieur à 0,6, la métropole paulistaine est ainsi un symbole à l'échelle urbaine de l'importance des inégalités socio-économiques du Brésil.

5 Les classes sociales aisées se concentrent dans certains quartiers. A la fin des années 80 , s'est amorcée la construction de centaines de grandes tours (condominiums), notamment destinées à une fonction résidentielle pour les catégories favorisées. C'est dans ce type de quartiers que résident les lycéens interrogés, au sud-est du quartier reconnu 
fonctionnellement comme étant le centre étendu de la mégapole. Le Lycée Pasteur est d'ailleurs également localisé dans cette zone, dans le quartier de Vila Mariana.

6 Si les élèves interrogés ne font pas partie des populations millionnaires de la cité paulistaine, il n'en demeure pas moins qu'ils appartiennent à la partie la plus aisée de la population de São Paulo, ce qui influence tant leurs représentations de l'espace urbain que leurs pratiques et mobilités dans la cité. Quelles sont les territoires du quotidien de ces adolescents en situation d'expatriation? Quelles en sont leurs représentations spatiales? Que disent-elles d'une métropole émergente comme São Paulo?

Figure 1: São Paulo : les divisions administratives

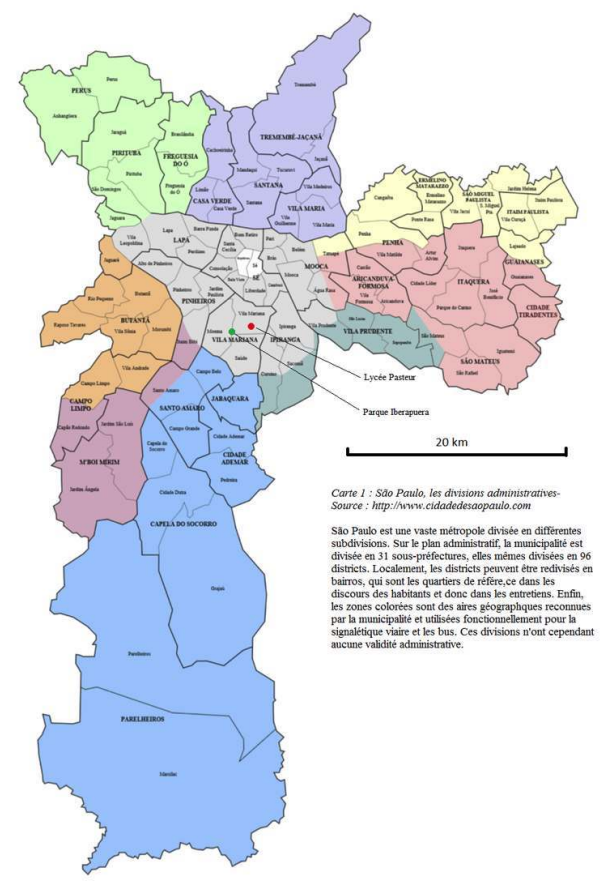

\section{Méthodologie}

7 Le procédé de la carte mentale est utilisé par les géographes depuis les années 1960 et l'émergence du courant de la " géographie de la perception » (Staszak in Lévy, Lussault, 2003). Les cartes mentales correspondent à des représentations subjectives de l'espace (Lynch, 1960). Il s'agit d'une représentation inexacte d'un espace vécu, mais sa distorsion est interprétée comme la transcription d'une représentation mentale. Ces perceptions sont soumises à des analyses qui permettent de définir des points de repère, des pratiques et des parcours citadins. La carte mentale est un outil, à la fois social, symbolique et sentimental nous permettant de nous représenter le monde mais aussi d'opérer des choix. Ainsi, les cartes mentales peuvent intervenir dans des processus comme la sélection de lieux pour le loisir, pour les achats ou pour la fonction domiciliaire.

Ces cartes transforment, valorisent ou déprécient un espace donné. Les cartes mentales ne sont pas statiques. Au contraire, elles sont dynamiques et évoluent selon l'âge, l'expérience, l'activité, l'emploi, les moyens financiers et le groupe social. Par 
conséquent, cette construction est continuellement refaçonnée non seulement par les individus mais aussi à travers les perceptions et représentations collectives d'un groupe social. Les cartes mentales révèlent les logiques de déplacement des citadins, les limites des quartiers, mais aussi les points stratégiques de l'espace dessiné, les points de repères et de références des usagers de la ville. Ces marqueurs de l'espace, entre autres, ont aussi été utilisés par A. Frémont pour définir la notion "d'espace vécu» (Lynch, 1960; Frémont, 1999). P. Gould et R. White se sont, eux, intéressés aux images mentales des individus pouvant expliquer leurs préférences pour un lieu, une région ou un pays. Par la suite, G. Di Méo a conceptualisé ceci en "espace imaginé » et en "espace de vie », ce dernier étant associé à « l'espace fréquenté et parcouru par chacun avec un minimum de régularité » (Di Méo, 1998).

Les deux géographes américains ont également eu recours aux cartes mentales pour mesurer la connaissance et l'ignorance des individus (Gould et al., 1984). Ainsi, en se servant de cartes mentales dessinées qui sont une représentation externalisée, on peut déduire une représentation interne. Nous avons eu recours aux « cartes mentales » avec cette méthode d'enquête nommée "sketch map » en anglais (que l'on peut traduire de manière plus précise par " carte à main levée ») et qui consiste à faire dessiner un espace par l'enquêté sur une page vierge. À grande échelle, celle de la ville, elles ont pour objectif d'étudier les itinéraires ou bien la manière dont l'individu se positionne dans l'espace. Il est donc question ici d'espace subjectif.

10 J'ai essayé de mettre à jour le rapport de l'individu à l'espace, comment il se positionne dans l'espace et comment il le perçoit.

11 Il a été demandé aux élèves interrogés en classe de dessiner de mémoire en 20 minutes « une carte de São Paulo » sur une feuille A4 sans précision de couleurs à proscrire. La consigne était large afin de permettre une liberté d'expression. Il n'y avait dans la salle de cours nul indice visuel de façon à éviter que les élèves puissent se repérer à partir de documents quelconques. La procédure avait pour objectif de faire établir de véritables cartes mentales individuelles et non des cartes mentales de groupe. Cependant, les élèves n'étant pas physiquement séparés de leurs voisins de table, on a noté de fortes similitudes sur certaines cartes. D'autre part, il est à noter que l'exercice de la carte mentale a été imposé aux élèves des deux classes de seconde. De fait, certaines productions lacunaires peuvent aussi l'être du fait d'une mauvaise volonté des élèves, la plupart s'étant cependant bien prêtés au jeu.

12 Les sentiments, les affinités, les craintes, les inclinations et les aversions ne peuvent pas raisonnablement être examinés seuls. Sonia Lehman-Frisch, Jean-Yves Authier et Frédéric Dufaux le rappellent dans leur étude du quartier de Batignolles vu par des enfants de CM1: "The drawings do not demonstrate the variable scope of the neighbourhood according to the children. Only the interviews allow to assess the contrasting expanse of their territory ..." (Lehman-Frisch, Authier, Dufaux, 2012, p27). Les cartes sont ainsi complémentaires du travail d'entretiens qui a aussi été mené auprès de quarante élèves de seconde, première et terminale. J'ai ainsi croisé les constats sur les cartes avec ceux issus d'entretiens semi-directifs réalisés auprès de lycéens dont le discours se nourrit d'expériences actuelles et/ou plus anciennes. La grille d'entretien constituée en amont portait sur les représentations, mobilités et territorialités des lycéens. Néanmoins, l'analyse doit aussi être manipulée avec beaucoup de précaution. En effet, les élèves interviewés ne représentaient qu'un effectif partiel des élèves du lycée Pasteur. Se pose alors la question de la représentativité des dires analysés. Il s'agit de trouver des 
constantes pour l'analyse sans nier la singularité des paroles entendues. Dans le mémoire, nous avons ainsi retenu les propos en fonction de la saturation des données qui est considérée selon le principe que « quand une hypothèse, par rectifications successives atteint un certain degré de pertinence, vient un moment où les données «empiriques diverses lui apportent une confirmation régulière» (Schwartz in Anderson 1993, p 286). Nous avons aussi tenté de restituer de manière la plus juste possible les différents regards portés par ces adolescents sur cette expérience de l'expatriation à « Sampa ${ }^{3}$ ».

L'analyse qui suit a permis d'obtenir des résultats: on note ainsi des distorsions de distances ainsi que la représentation affective et sensorielle de l'espace. Ces représentations permettent aussi de rendre compte d'un espace non situé et sursémantisé. L'espace paulistain s'affranchit du réel (distance, localisations) pour devenir une image d'espace tel qu'il est perçu et ainsi contribuer à une iconisation spatiale.

\section{Raconte-moi où tu vis}

\section{Limites de la ville et (im)précisions des localisations}

Les cartes mentales ont été analysées selon les critères qui suivent. J'ai tout d'abord identifié les limites de chacune des 47 cartes mentales et leurs couvertures de surface ont été mesurées. Toutes les cartes mentales ont ensuite été comparées et triées en termes de type et de complexité fondés sur les réseaux de communication (axes structurels), les points de communication (carrefours, giratoires, intersections), les immeubles et autres repères de taille, les espaces non construits (parcs, cours d'eau...), les limites et les fonctions afin d'aboutir aux résultats ci-dessous.

15 La région métropolitaine de São Paulo, en portugais Grand São Paulo, réunit 39 municipalités de l'État de São Paulo constituant une conurbation. Elle englobe plus de 20 millions d'habitants, c'est la troisième plus grande aire urbaine du monde. En ajoutant à cette région métropolitaine les régions métropolitaines proches (Campinas, Baixada Santista, São José dos Campos, Sorocaba, Jundiaí), on obtient le complexe métropolitain étendu de São Paulo-Campinas qui dépasse 29 millions d'habitants (environ $75 \%$ de la population de l'État). Ce complexe forme déjà la première métropole de l'hémisphère Sud avec 65 municipalités et $12 \%$ de la population brésilienne.

Pour les élèves, parler de São Paulo revient à prendre en compte la municipalité de São Paulo et non la région métropolitaine. Comme on peut le voir plus haut (Figure 1), la municipalité de São Paulo est divisée en 31 arrondissements (subprefeituras), groupés dans neuf zones (zonas). Or, si l'on regarde l'échelle des cartes des élèves, on se rend compte que les cartes vont de la représentation de la vue du balcon de l'appartement à la représentation de plusieurs quartiers. En revanche, aucune carte ne représente l'intégralité de l'agglomération de São Paulo (superficie et nombre de quartiers) et aucune ne fait figurer les favelas. Outre la taille de la municipalité $\left(1523 \mathrm{~km}^{2}\right)$ cette occultation correspond à des pratiques très localisées. Les cartes qui représentent les superficies les plus vastes puisque qu'elles font figurer l'aéroport de Congonhas ne représentent pas les espaces interstitiels entre le centre de la métropole et l'aéroport et se rapproche davantage de la topologie que de la carte (Figure 2). 
Figure 2 : Production graphique de S. élève de seconde au Lycée Pasteur

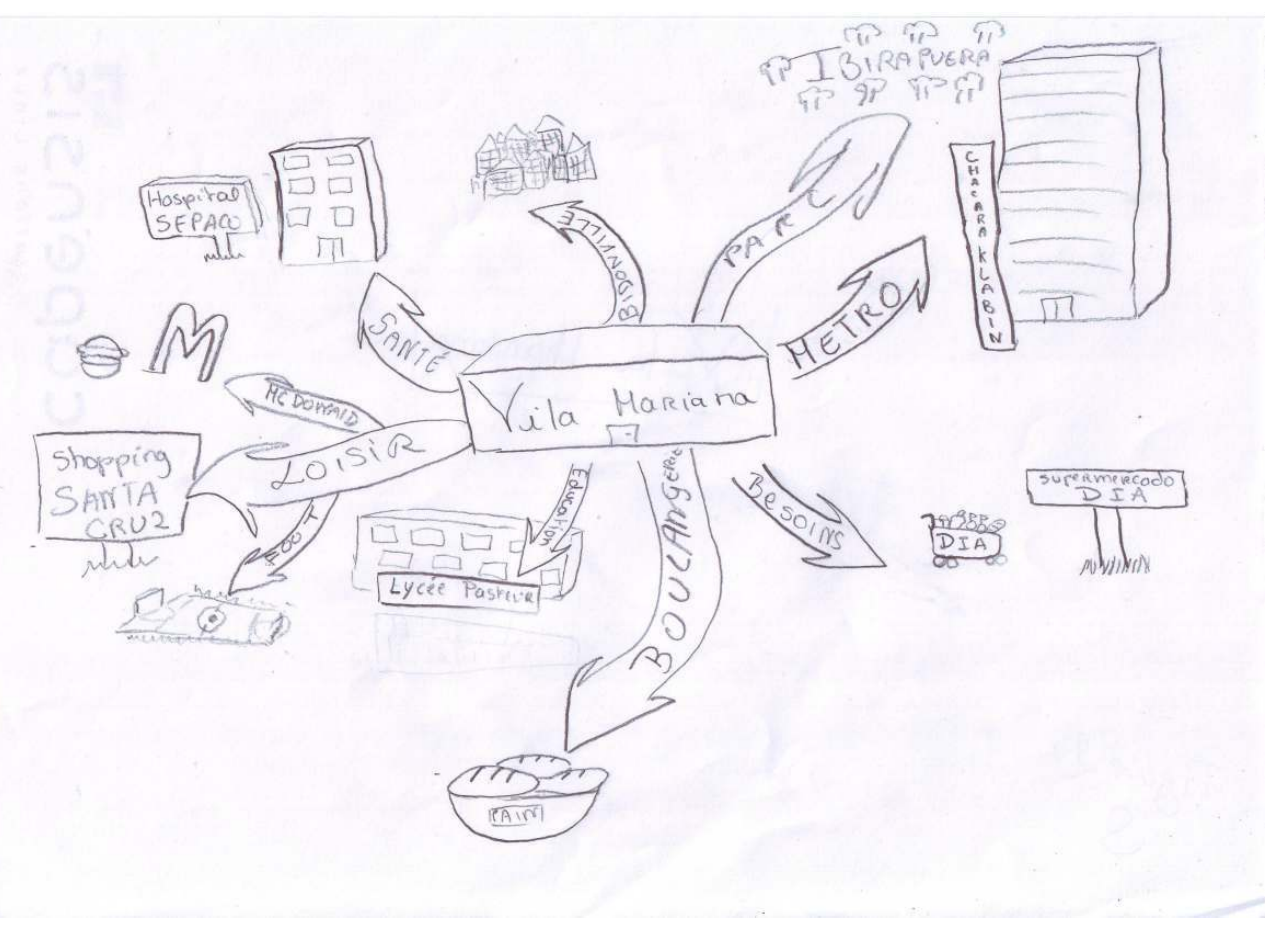

São Paulo, année scolaire 2014

Une typologie a ainsi été établie de la couverture de surface la plus petite (constructions individuelles) à la couverture la plus large (municipalité + aéroport) :

- du balcon au micro-quartier, $19 \%$ des cartes mentales (Figure 3),

19 - de un à trois quartiers, 51\% (Figure 4),

20 - São Paulo, 30 \% des cartes mentales (Figure 5). 
Figure 3 : Production graphique de L. élève de seconde au Lycée Pasteur

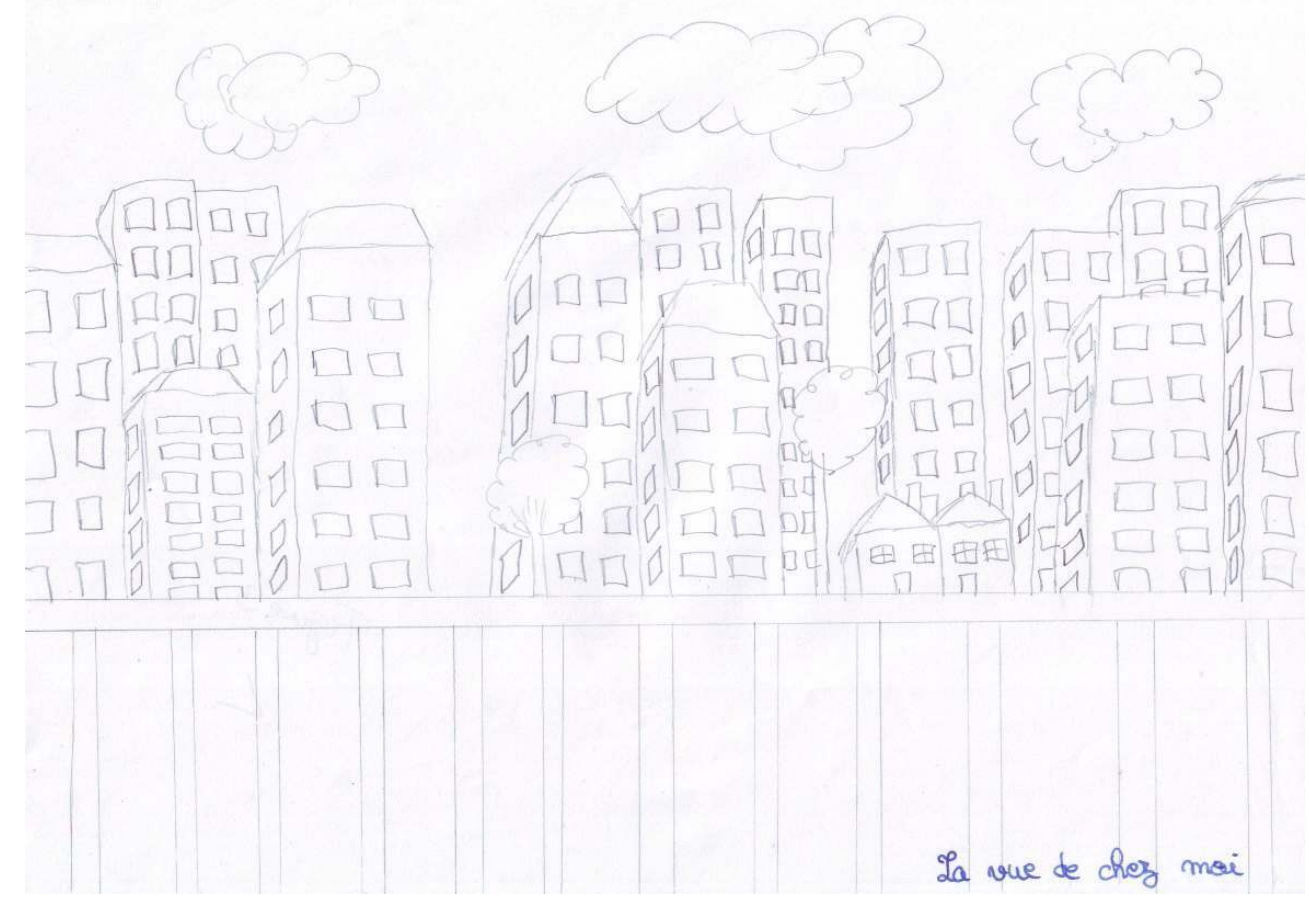

São Paulo, année scolaire 2014.

Figure 4 : Production graphique de V. élève de seconde au Lycée Pasteur

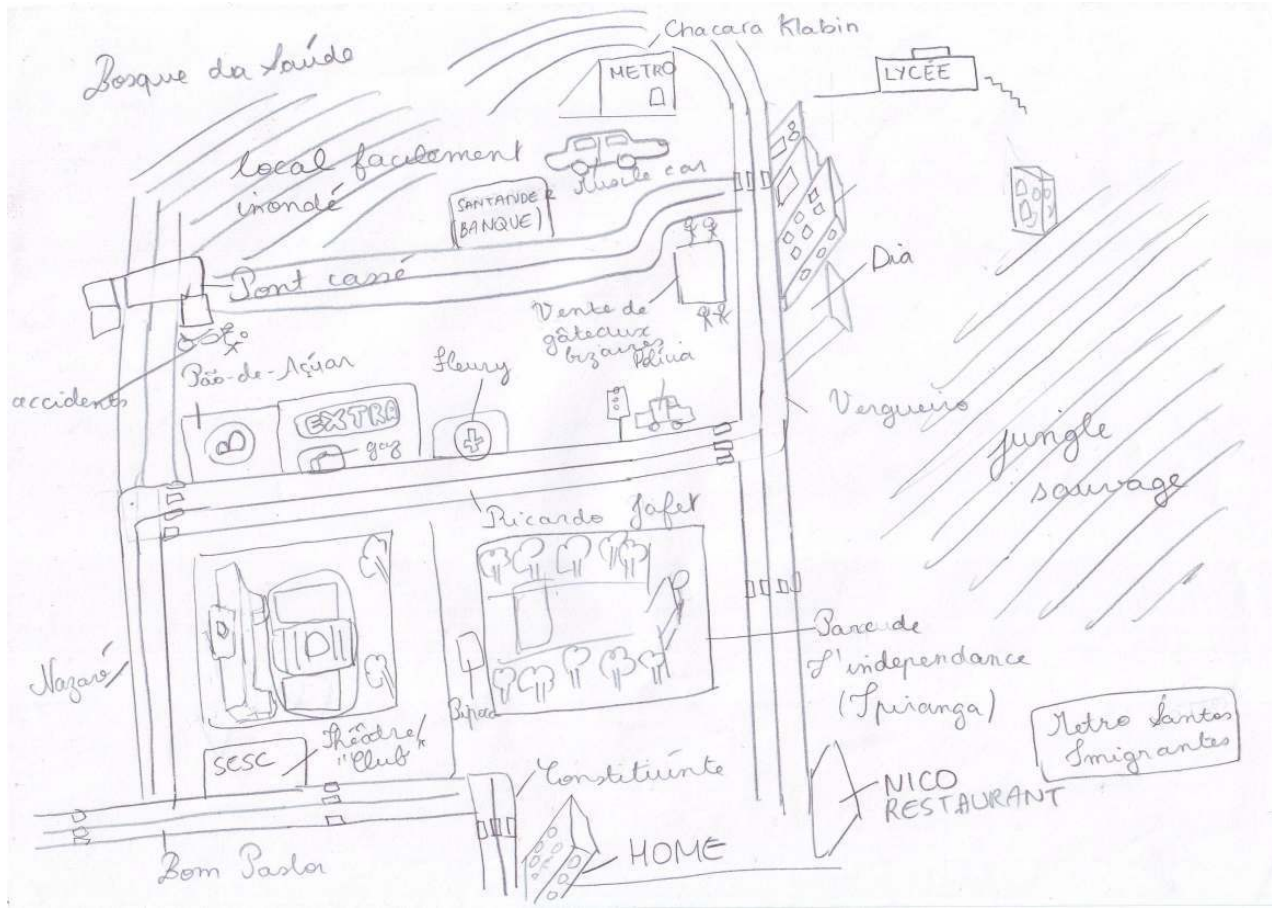

São Paulo, année scolaire 2014. 


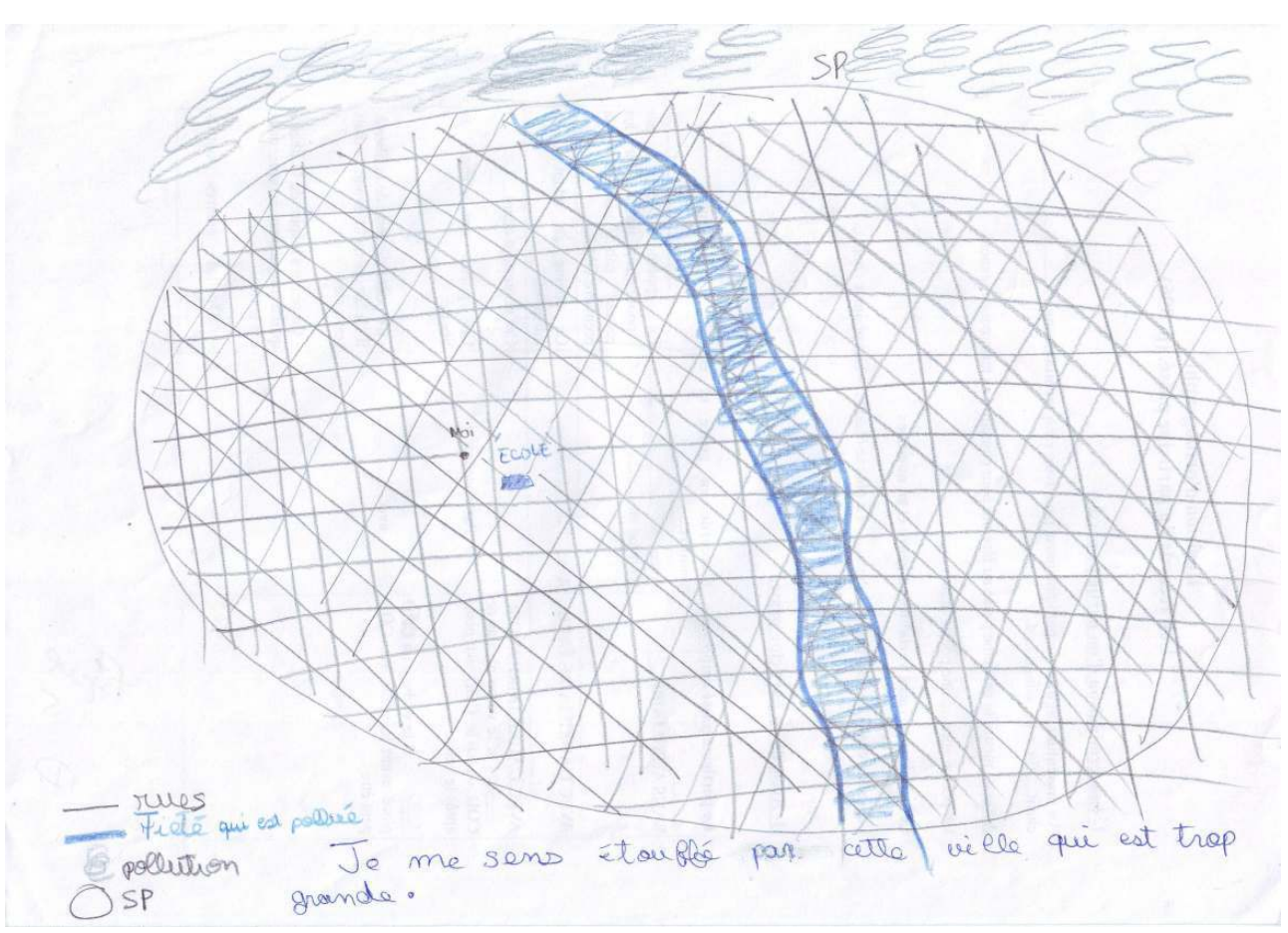

São Paulo, année scolaire 2014 couverture de surface et/ou d'index de détail. On a pu constater que les différences sont étroitement reliées à la durée de séjour à São Paulo. De manière générale, les élèves qui sont dans la ville depuis plus longtemps dessinent des cartes mentales avec des couvertures de surface beaucoup plus grandes ou beaucoup plus détaillées et beaucoup plus précises que celles confectionnées par les élèves qui sont arrivés depuis moins d'un an.

Si l'on raisonne uniquement en termes de capacité à identifier les détails, on se rend cependant compte que toutes les cartes mentales présentent les mêmes lacunes : peu de noms de quartiers et d'axes (hormis la Paulista), pas d'orientation. L'espace paulistain apparait dans ces productions comme un espace symbolique, s'affranchissant de la logique situationnelle. Les quelques noms précis ne sont pas placés en fonction de leur situation réelle mais cités à titre illustratif des loisirs pratiqués par les élèves. Ils sont ainsi placés sur les productions pour leur valeur affective et non en tant que points ou espaces fixes dans l'espace. Cette difficulté de perception de l'espace de la ville peut en partie s'expliquer par le fait que les élèves ont peu l'occasion de parcourir la ville en bus ou à pied.

$\mathrm{J}$ : « C'est difficile de marcher dans les rues, de se balader, contrairement à la France où on peut se balader. Là on marche dans la rue que quand on est obligés. »

M: « Je ne connais pas trop les quartiers parce que je donne juste l'adresse au taxi et il cherche.»

F: « Quand j'étais à Paris je sortais à pied, en bus ou en métro. Surtout à pied, même à $3 h$ du mat. Ici on ne peut pas faire ça. » 
Figure 6 : Production graphique de B. élève de seconde au Lycée Pasteur

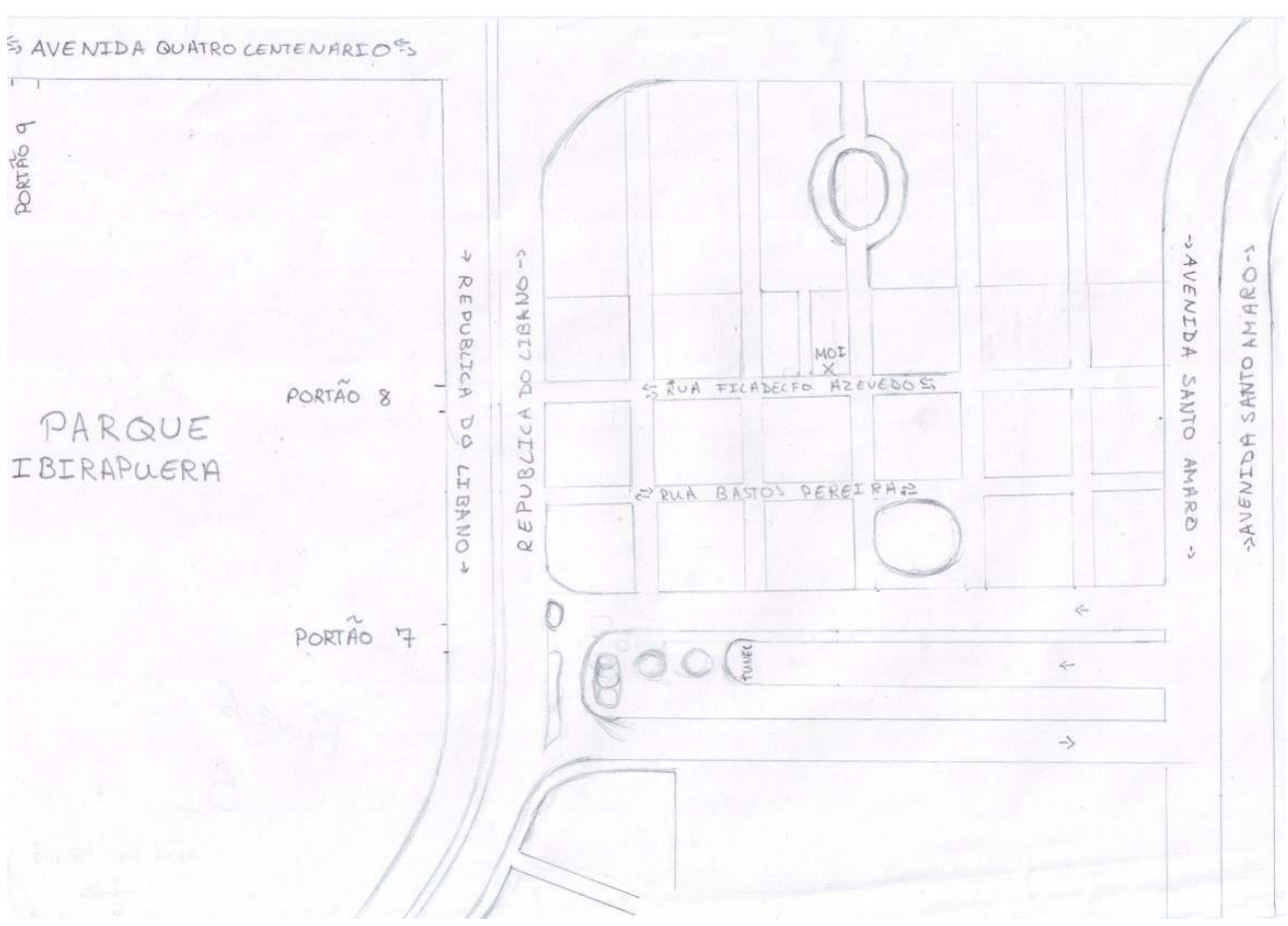

São Paulo, année scolaire 2014

La taille réduite des aires couvertes dans les cartes mentales montre très clairement des pratiques spatiales restreintes et une territorialité marquée. Cela correspond dans les entretiens à un manque de mobilité et une consommation de services et de loisirs sélectionnés. Comme précédemment évoqué, une partie des élèves a dessiné des cartes mentales à l'échelle du micro-quartier $(<0,5 \mathrm{~km} 2)$ voire la vue de São Paulo depuis chez eux, ce qui reflète une exploration spatiale restreinte due à des pratiques fondées sur le " chez soi ». Les élèves se retrouvent chez leurs camarades de lycée mais ceux-ci demeurent majoritairement dans les mêmes quartiers comme en témoigne la carte ci-dessous (Figure7). On note en effet sur la carte réalisée à partir des adresses des élèves fournies par le Proviseur du lycée que les familles d'expatriés habitent majoritairement autour du parc Iberapuera, ce qui ressort aussi des entretiens. Le lycée se situe quant à lui dans le quartier de Vila Mariana (cf Figure 1).

$\mathrm{V}$ : « En fait les expatriés restent dans leur coin. Ils ne bougent pas trop. On change de quartier, mais en fait c'est toujours les mêmes quartiers et c'est toujours des quartiers privilégiés. Tout ce qui est dans la zone nord ... on n'y va pas. En fait la plupart des expatriés restent dans la zone sud qui est la zone la plus riche. Tout ce qui est au nord et à l'ouest, les quartiers qui craignent un peu, on évite d'y aller. » 


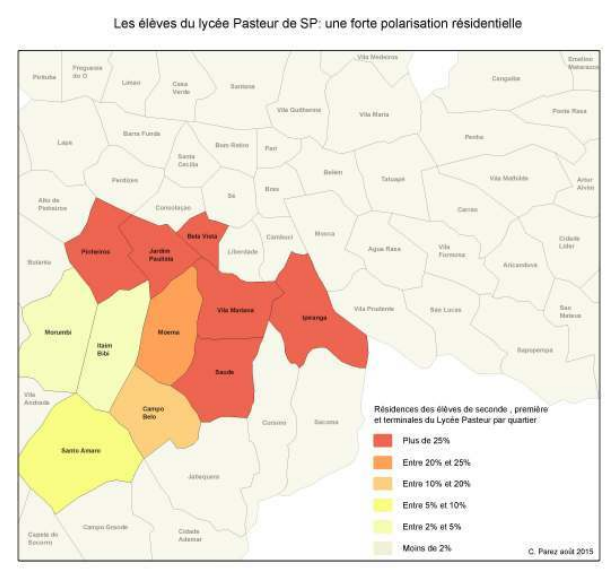

On doit donc prendre des précautions quant à l'interprétation des espaces vides sur les cartes mentales. Sont-ils totalement inconnus, peu connus ou délibérément non dessinés ? Dans le cas présent, il semblerait que certains lieux ne sont pas dessinés parce que dans l'esprit des adolescents, ils sont perçus comme dangereux ou redoutés, non pratiqués et n'appartiennent donc pas au São Paulo des élèves interrogés lors de ce terrain.

L: « Moi je préfère ne pas avoir conscience des favelas, ne pas avoir conscience que ça existe parce que ça me met vraiment mal à l'aise. Donc je préfère oublier que ça existe ... » $\mathrm{P}:$ «Il faut éviter d'aller dans les favelas. C'est dans ces parties de la ville qu'il y a les gens les plus violents. C'est à éviter je pense. »

\section{Une métropole standardisée}

Si l'on analyse les 47 cartes mentales de São Paulo pour saisir comment les adolescents français expatriés organisent et représentent leur espace vécu par ce qu'ils dessinent, on aboutit aux conclusions suivantes : $49 \%$ d'entre eux organisent leur espace à partir des rues et autres artères, $31 \%$ l'organisent à partir de repères (construits ou non construits, château, parcs), $20 \%$ ne font apparaître aucun repère spatial. Sur ces productions, la métropole paulistaine est une cité d'immeubles sans trame définie, sans nom de rues, sans limite précise à la ville qui est représentée comme une " jungle d'immeubles ». Dans cette série de dessins, l'espace paulistain, dont l'imprécision de la trame viaire est manifeste, apparaît comme un espace où l'architecture de condominiums est perçue comme étouffante. Les cartes mettent ici en évidence une métropole standardisée, à l'architecture verticale de condominiums (Figure 8), dont toutes les rues seraient 
similaires, une vision influencée par le fait que les élèves interrogés résident massivement dans le sud est du centre étendu.

Figure 8 : Production graphique de M. élève de seconde au Lycée Pasteur

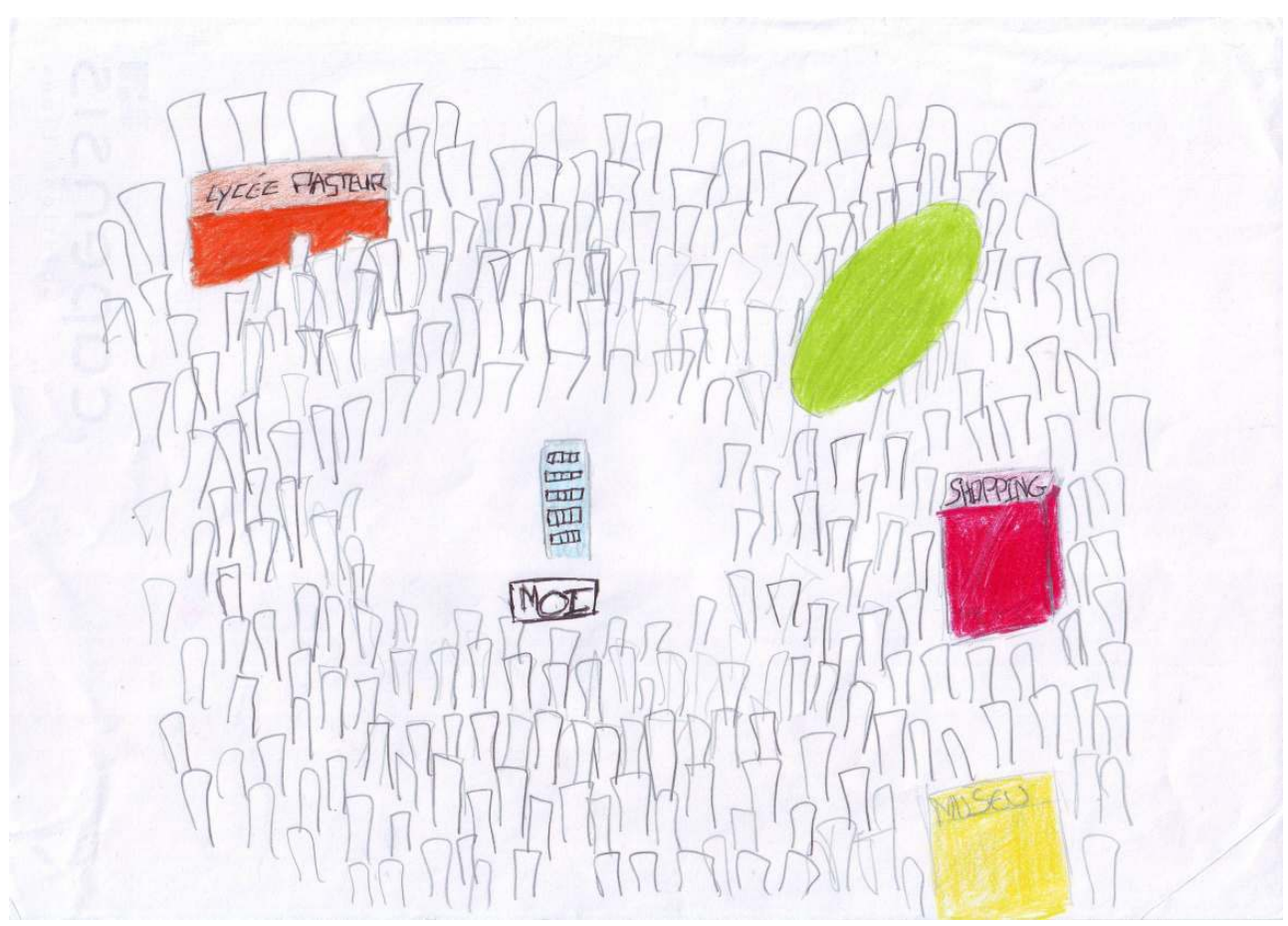

São Paulo, année scolaire 2014

\section{Un rapport affectif à la ville}

A ce stade de l'analyse, on se rend ainsi compte que pour ces adolescents, représentation spatiale et pratique spatiale sont très liées. De nombreuses cartes mentales de ces adolescents identifient des lieux précis qui, dans les entretiens sont chargés d'affectivité (magasins, maison, cinémas, facilités sportives et récréatives, école, ambiance et parcs urbains).

L: «Je ne trouve pas que ce soit beau parce qu'il y a beaucoup d'immeubles. Tout est gris. Il y a de bons aspects de São Paulo par exemple les bars, le parc, les shoppings, les cinémas sont très bien et c'est le Brésil quand même : il y a le climat, la plage, la nourriture brésilienne, tous les fruits donc même si São Paulo ce n'est pas l'image idyllique du Brésil, ça réunit quand même tous ces critères, c'est déjà bien même si ce n'est pas une ville que j'apprécie beaucoup. » 


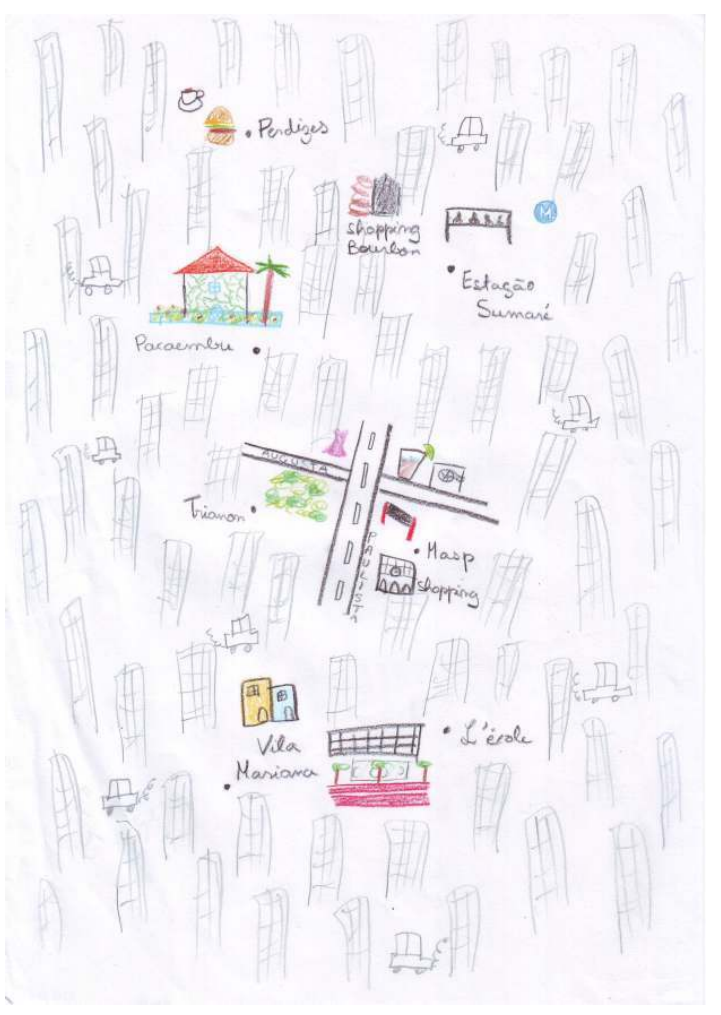

São Paulo, année scolaire 2014

En parallèle, j'ai d'ailleurs noté que le vocabulaire utilisé dans les entretiens pour désigner la ville et ses espaces est aussi celui de l'affectivité : «j'aime, je déteste, j'adore ... ».

T: «J'aime beaucoup São Paulo je trouve que c'est une ville qui a un grand potentiel. Je préfère à Rio. »

L : « Moi j'adore« São Paulo. J'aime bien la ville. Il y a beaucoup de Français qui n'aiment pas du tout, qui trouvent que c'est trop bétonné, trop d'appartements mais moi ça ne me dérange pas. »

Le facteur affectif est aussi présent sous son volet répulsion via le rejet que peut susciter la métropole paulistaine représentée comme un espace anonyme et anxiogène.

K: « Quand je suis arrivée j'ai pleuré parce que j'ai trouvé que la ville est juste affreuse ! » $\mathrm{R}$ : «Cette ville, je ne l'aime pas du tout. Les gens sont sympas mais la ville, je ne l'aime pas. J'ai vécu toute ma vie dans des grandes villes et là vraiment, il y a trop de pollution. J'ai envie d'une petite ville, plus calme, où les gens seraient moins stressés. Là il est temps de changer."

D: « C'est laid, c'est ignoble, c'est moche. Il n'y a pas de style. Il y a un truc qui sort qui a un genre, puis un autre truc qui a aucun genre. Trop de fils électriques. Des grands parcs il y en a que deux. Par contre il y a plus d'arbres qu'à Paris. Mais à Paris des espaces de verdure il $\mathrm{y}$ en a quand même plus au sens où il $\mathrm{y}$ a des petits squares où on peut s'asseoir.»

M: « Je suis allé dans plein de villes. Sampa c'est des buildings et des pâtés. Ça a pas de charme. Après on peut dire qu'il y a du charme par rapport à ce qu'il s'y passe, à l'intérieur. Les gens sont sympathiques, tout ça mais si on regarde juste sur google earth, 
c'est nul. Après il y a quand même beaucoup d'arbres ... C'est ça São Paulo: des arbres, des immeubles, des favelas. »

De cette dimension affective du rapport à l'espace, des éléments à caractère morphologique émergent aussi de cette étude. Ils permettent de considérer São Paulo dans le discours de ses adolescents comme une allégorie urbaine, à la fois «non lieu » et «hyper lieu» en tant que norme de la métropole anxiogène dans les représentations adolescentes.

\section{La «forme d'une ville » : représentations de la morphologie urbaine paulistaine :}

\section{La question des centralités} administratif de Sé est composé de neuf districts. Cet espace a connu son apogée résidentielle et fonctionnelle dans les années 1930-1950. Oscar Niemeyer y a ainsi dessiné des immeubles, sur la Praça da República. Depuis la fin des années 1970, ce centre perd des habitants; ceux qui restent sont dépourvus de moyens, vieillissent, nombre d'immeubles sont abandonnés, parfois squattés et les commerces informels y sont légion. En revanche, les lignes de transport en commun, les nombreux commerces parfois traditionnels et les petits établissements témoignent de l'espace de ressources que peut constituer le centre. Ce centre a bénéficié de l'action volontariste de l'Etat et de la municipalité qui se sont largement engagés pour sa revitalisation, en y installant des bâtiments publics et en favorisant de nouvelles créations architecturales. Cependant, il reste réputé peu fréquentable, la nuit en particulier. Au sud-ouest s'élèvent des business centers, emblème du fonctionnalisme urbain contemporain, associés à des shoppings centers de standing. C'est l'exemple du quartier d'affaires de Berrini, trente ans après que l'Avenida Paulista a supplanté dans les années 60 ce que l'on qualifie aujourd'hui de centre historique (Sé et República). Le centre historique paupérisé est connu des élèves, parfois représenté sur les cartes mais n'est que très peu un espace visité par eux-mêmes et leurs familles. Le Centro est ainsi reconnu par certains pour sa signification historique et architecturale mais manque d'attractivité.

G: « Le centre historique c'est le fouillis, je n'aime pas du tout. Souvent le centre c'est une partie de la ville qui est très belle. Ici ça fait un peu miteux, c'est pas très beau. »

S: « Le centre historique, on n'y va pas c'est différent de la France. Il pourrait être beau alors que là il n'est pas entretenu. Et on m'a dit que c'est dangereux, tout seul c'est dangereux, la nuit surtout. C'est à côté de chez moi, je pourrais y aller mais je n'y vais pas. »

R: « Il y a un centre-historique, la Sé tout ça mais bon ... »

$\mathrm{T}$ : « Moi quand je vais dans le centre, c'est plutôt pour des expositions mais mes parents n'aiment pas trop que j'y aille parce que c'est dangereux. Quand il y a des concerts la nuit, ils ne me laissent pas y aller. »

$\mathrm{Y}$ : «Je pense que la préfecture veut revaloriser le centre donc il y a pas mal d'actions culturelles là-bas mais il n'y a pas beaucoup de monde qui y va. Sinon c'est bien. Moi j'aime bien l'architecture. Il y a un mélange. Le début de la création de SP et à côté il y a de grands buildings. Moi j'adore ça.

Carnets de géographes, 10 | 2017 
V: « Moi j'y vais souvent dans le Centro. On peut y acheter des bijoux, des tissus ... C'est juste que c'est un peu dangereux parce qu'il y a beaucoup de monde alors il faut être encore plus attentif mais si tu y vas sans bijoux très cher, tu peux y aller sans aucun problème. En fait il y a beaucoup de monde, surtout des Brésiliens assez pauvres et beaucoup d'immeubles squattés et du coup les gens n'aiment pas trop, ça fait un peu peur.»

M: « C'était le plus beau quartier avant. C'était le centre vraiment avec de magnifiques banques et tout. Le problème c'est qu'ils l'ont laissé se détériorer. Par exemple ma grandmère -je suis franco-brésilienne- elle m'a raconté qu'avant c'était un très beau quartier et que c'était très bien mais maintenant il y a par exemple des mendiants qui urinent dans la rue. Mais il y a des choses à voir, l'église de la Sé par exemple.

Le centre historique n'est ainsi représenté sur les cartes mentales qu'à une seule occasion. La ville de São Paulo apparaît dans ces représentations comme apatrimoniale et la question de la centralité, associée en Europe au centre historique y est donc pensée différemment. Si un centre de la métropole est parfois représenté, il peut de prime abord apparaître comme étant l'avenue Paulista, l'artère principale, symbole de la métropolisation paulistaine, ce que les entretiens viennent confirmer. La Paulista (Bela Vista-Jardim Paulista) est bien représentée et perçue comme quartier: importance architecturale, boutiques, bars localisés sur cette artère et constituant un axe à gros trafic (Figure 10).

$\mathrm{V}$ : «Ce n'est peut-être pas joli mais quand on est sur la Paulista c'est impressionnant. Je l'aime cette ville. Quand je suis arrivée, j'ai pourtant détesté tous ces immeubles. » $\mathrm{H}:$ «Je dirais que le centre c'est la Paulista. »

De fait, les lycéens conçoivent davantage São Paulo comme une métropole polycentrique et c'est pour eux l'un des critères majeurs de différenciation avec l'espace urbain européen pour lequel l'identité de la ville repose bien souvent sur le patrimoine architectural du centre historique. 
Figure 10 : Production graphique de U. élève de seconde au Lycée Pasteur

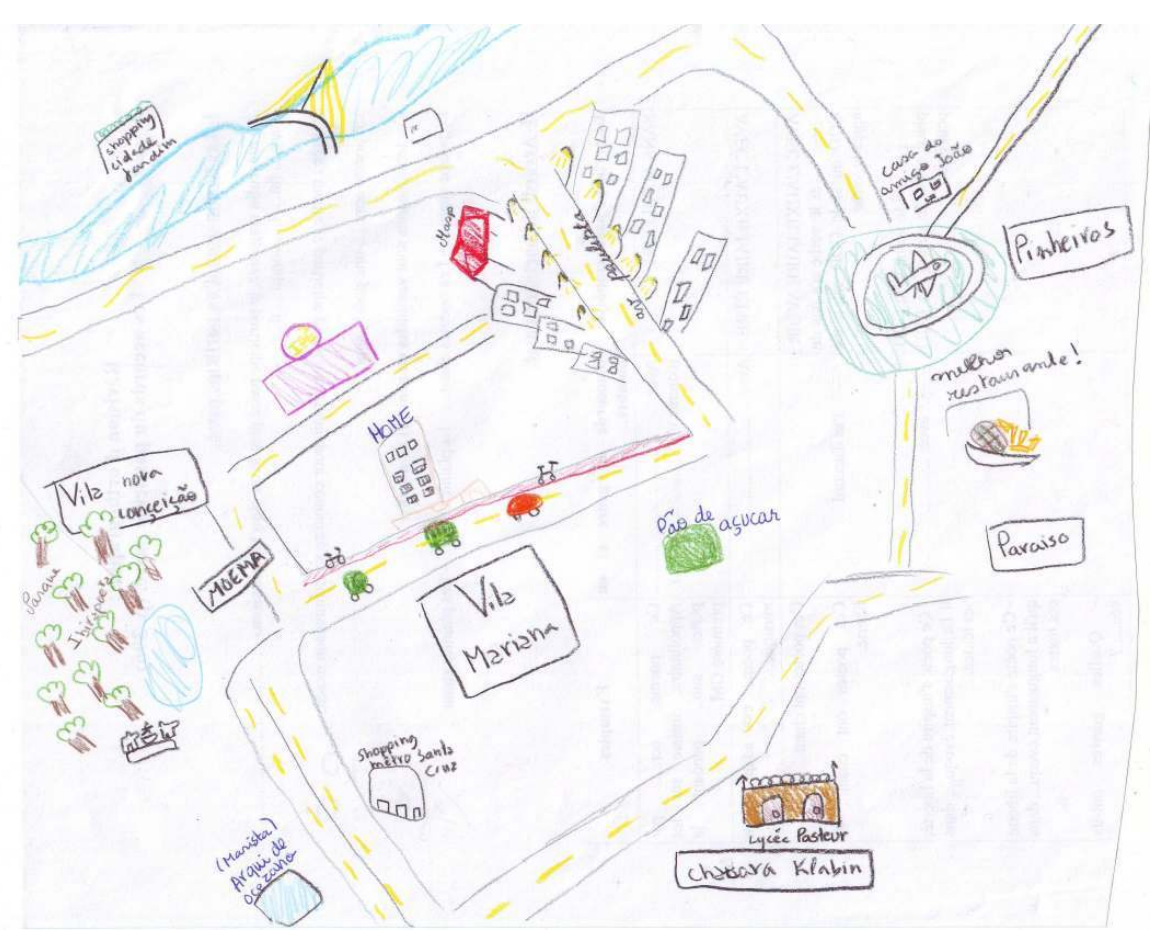

São Paulo, année scolaire 2014.

$\mathrm{P}:$ « Il n'y a pas vraiment de centre-ville ici donc ça ne donne pas trop envie de boire un verre. En Europe on dit « on va dans le centre » et on ne sait pas ce qu'on va faire mais on dit « on va dans le centre » alors qu'ici on ne peut pas aller dans le centre vu qu'il n'y en a pas ici donc c'est vraiment un mode de vie différent. »

$\mathrm{L}$ : « Il y a le Centro, le centre historique. Après pour ce qui est culturel tout ça c'est Vila Madalena et sinon Jardins Iberapuera qui peut être un centre. C'est tellement grand ... Le centre historique avant je faisais de la peinture là-bas mais on n'était pas rassuré quand on sortait de la voiture.»

0 : « Le centre pour moi c'est là où on va le plus donc pour moi ce serait Jardins, Iberapuera.»

I: « En fait Sampa c'est tellement grand qu'il n'y a pas un seul centre. Pour les centres ce serait plutôt des « centres d'intérêt ». Il y a le centre où l'on se retrouve pour aller manger, celui où l'on se retrouve pour aller faire les boutiques ... »

Un autre exemple de centralité, qui confirme par ailleurs le rapport affectif à l'espace, est le parc Iberapuera. Très présent dans les productions, le parc est pourtant placé de manière aléatoire. Cependant, sa taille toujours conséquente correspond bien à la valeur affective que les élèves lui attribuent dans les entretiens.

L: « Le parc Iberapuera c'est le Brésil qui me plaît. Un Brésil qui est propre, beau et agréable. Qui est bien pensé ... ce qui est surprenant. Une architecture bien pensée dans ce pays ...»

En termes de repère spatial, même s'il n'est pas précisément situé, le parc Iberapuera apparaît comme un point de référence majeur pour les élèves. Nombre d'entre eux vivent à proximité de ce parc qui est d'autre part un espace de loisirs, de sorties familiales et amicales. 

est alors intéressant de noter que si les élèves ont souvent dessiné en noir et blanc, dans $75 \%$ le parc Iberapuera est représenté des cas en vert alors que le reste de la production graphique ne fait pas nécessairement apparaître d'autres couleurs symboliques (Figure 11).

D'autre part, le parc est également considéré comme le "poumon» d'une ville caractérisée par la grisaille et la pollution. En rupture avec cette grisaille souvent citée et dessinée, le parc Iberapuera est alors un centre de la mégapole mais aussi emblème du Brésil considéré comme un espace que les élèves qualifient de «tropical ».

M: « Je me rappelle quand on est arrivés, quand on était dans le taxi. L'un de mes petits frères- ils sont jumeaux- a dit : « Oh c'est moche ! ». Il y avait plein d'immeubles gris avec des graffitis et des tags et nous on n'aime pas trop ça les tags et les graffitis tout moches, lui il aime encore moins. Juste l'aspect, on n'aimait pas du tout. Par contre ce qui est bien c'est qu'il y a tellement de verdure que on se sent limite au milieu d'une forêt parfois. Par rapport à Paris, il y a dix fois plus d'arbres. Ça je trouve ça super chouette. »

Figure 11 : Production graphique de Q. élève de seconde au Lycée Pasteur

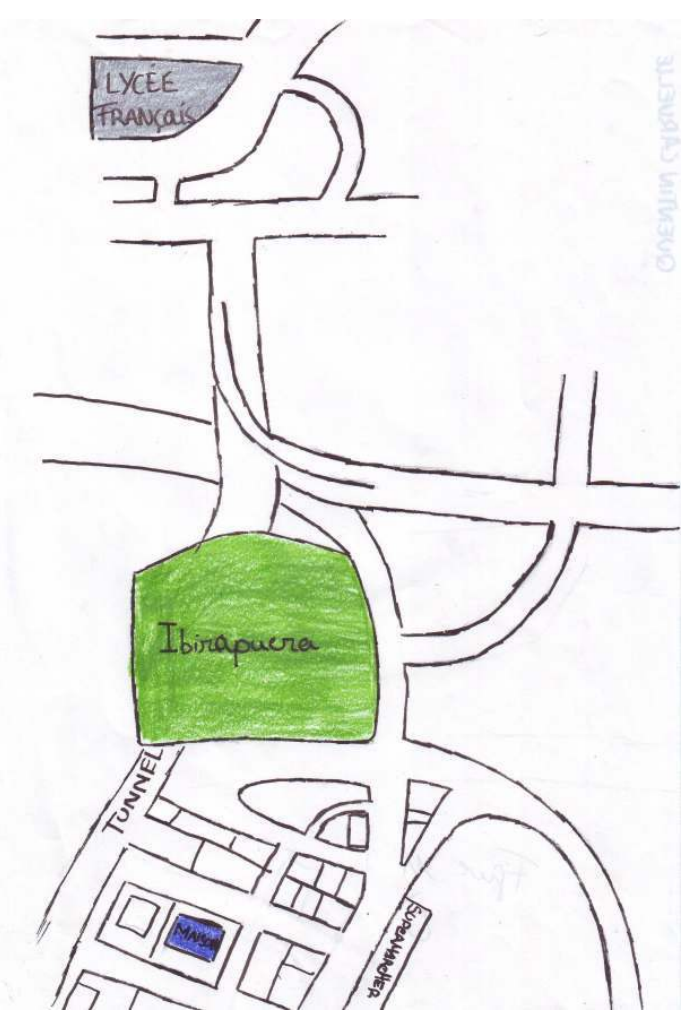

São Paulo, année scolaire 2014

\section{La ville de l'automobile}

Un autre critère de différenciation par rapport aux espaces urbains européens dont ces adolescents sont familiers est donc aussi l'omniprésence de l'automobile au détriment du piéton. Une production note ainsi les distances temps en voiture. A ce titre, on retrouve un élément récurrent dans les entretiens : l'importance de la durée des transports qui prime sur la distance effective et les difficultés de circulation (Figure 12). 
$\mathrm{H}:$ « C'est une ville très grande, $c^{\prime}$ est parfois difficile de faire des choses. Par exemple par rapport au trafic, ça peut prendre 20 minutes et le lendemain 40 . On ne sait jamais, ça c'est un vrai problème. Surtout que moi je fais beaucoup de déplacements en voiture. En fait ça dépend vraiment de l'endroit où on habite. »

Figure 12 : Production graphique de B. élève de seconde au Lycée Pasteur

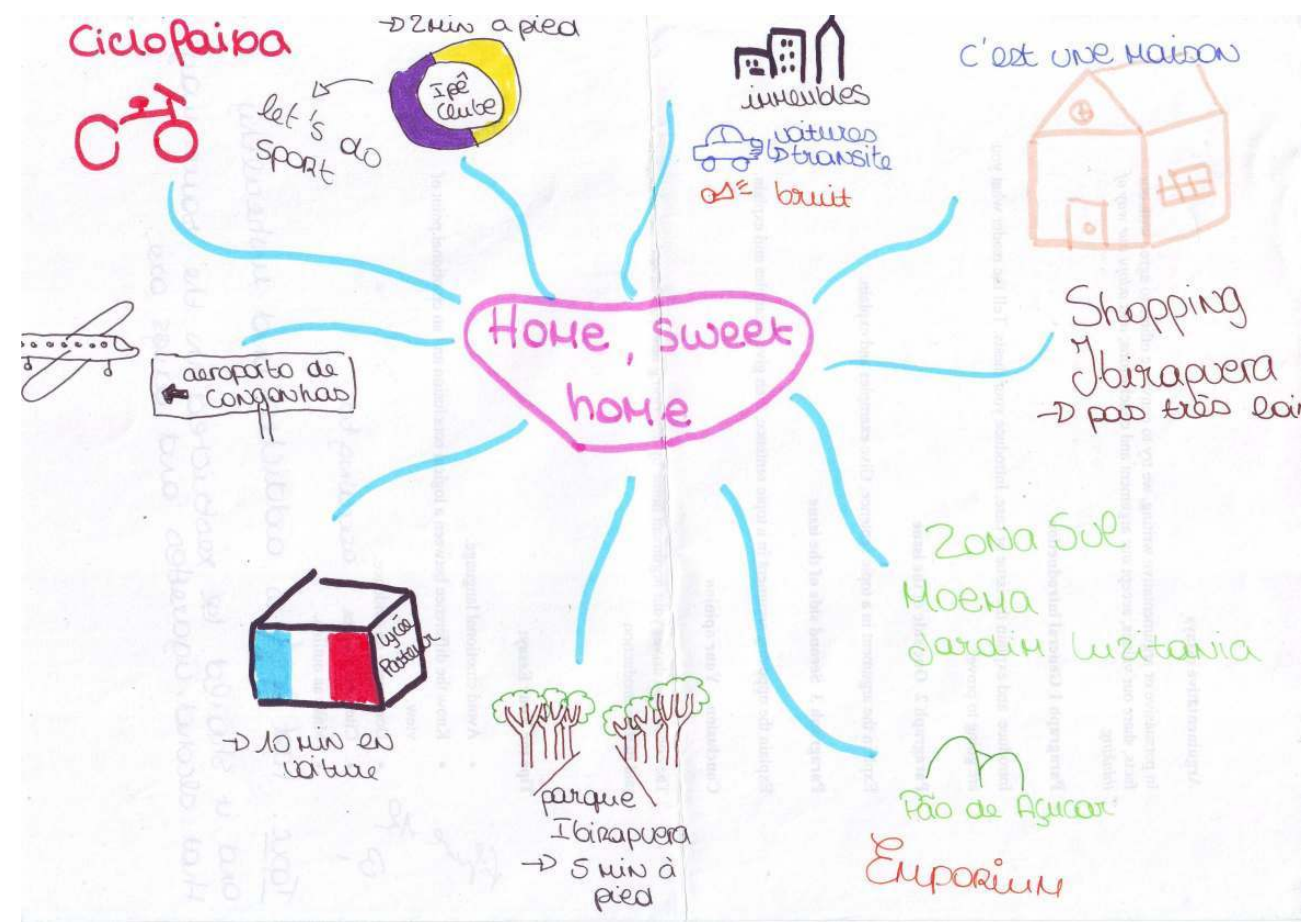

São Paulo, année scolaire 2014

H: « Ici c'est pas très bien organisé. Il y a toujours des embouteillages. Les rodizios, c'est l'alternance selon les numéros de plaques et les jours, ça marche pas très bien. C'est pas possible de rentrer chez soi le soir sans avoir deux heures d'embouteillages et ils peuvent mieux faire en matière de transports, c'est rien comparé à la France. »

$\mathrm{P}:$ « São Paulo est très polluée. La plupart des problèmes de Sampa sont en fait des problèmes communs au Brésil de manière générale comme les inégalités et en plus des problèmes qui sont liés à la ville même : la pollution, des problèmes de trafic. Ça arrive qu'il y ait 500 à 600 kilomètres de trafic dans la ville de São Paulo. Ce sont des chiffres qui atteignent le niveau national en France. Il y a beaucoup de trafic à São Paulo. São Paulo manque de transports en commun, il y a trop de trafic et trop de pollution mais les trois choses sont liées. Si on parvient à avoir plus de transports en commun, il y aura moins de trafic et moins de pollution donc ça pourrait se régler.»

Sur les productions graphiques, la présence des automobiles est ainsi récurrente, lesquelles dégagent toujours un nuage de fumée. Elle est considérée par ces jeunes comme l'un des maux urbains majeurs de cette ville. La pollution est représentée sur les cartes par la grisaille mais aussi par des automobiles expulsant des gaz d'échappement voire par le substantif "pollution » écrit tel quel sur les productions. Elle est ainsi représentée dans $56 \%$ des cas, voire est écrite sur $20 \%$ des cartes et est citée comme problème de São Paulo dans $80 \%$ des entretiens. 
Figure 13 : Production graphique de S. élève de seconde au Lycée Pasteur

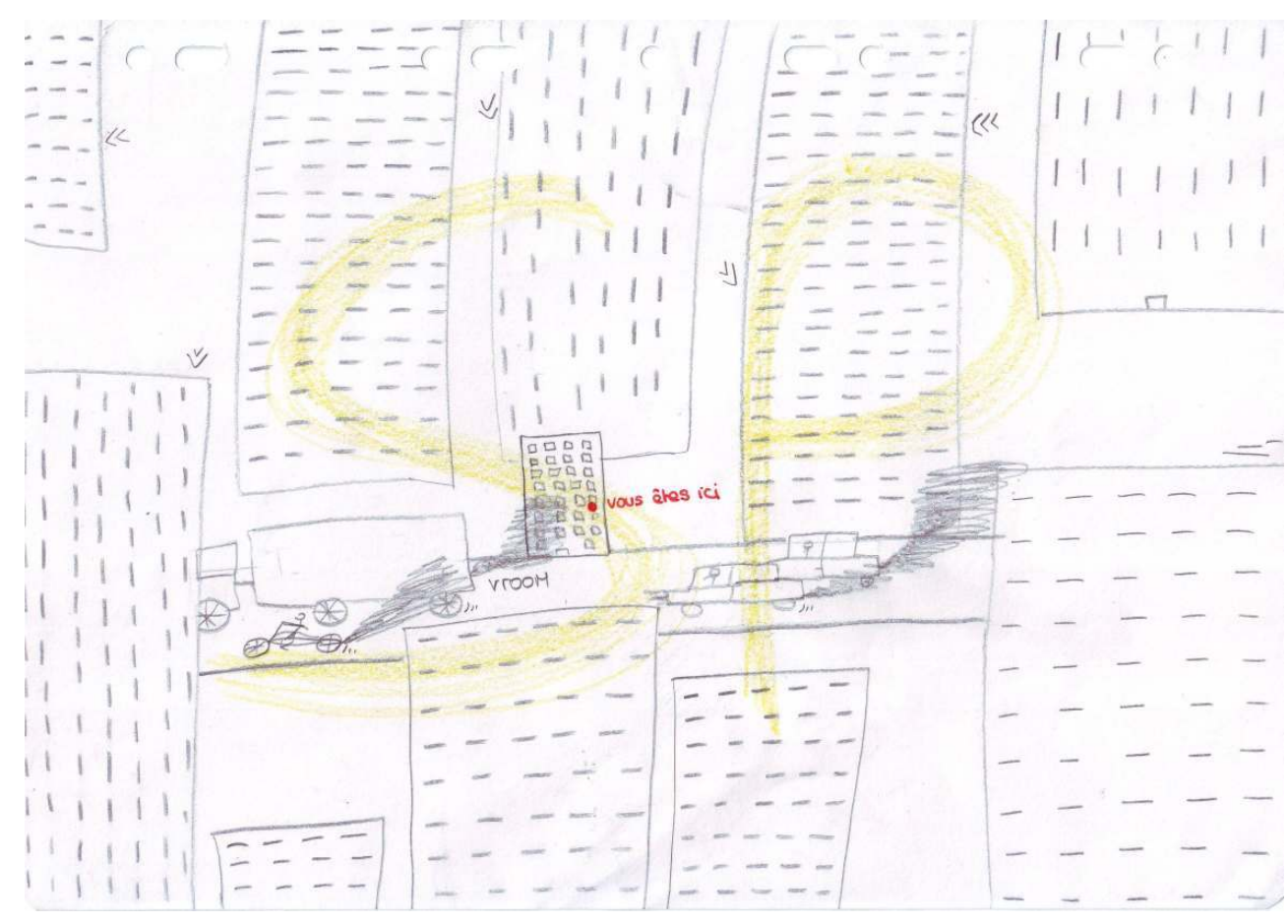

São Paulo, année scolaire 2014.

On a ici affaire à des représentations qui mobilisent la perception visuelle avec l'utilisation de la couleur grise sur les cartes pour représenter le constant nuage qui plane sur la métropole (Figure 13). On note également des références à la perception olfactive ainsi qu'à la perception auditive.

$\mathrm{V}$ : «C'est pas très joli, c'est sale. Et la pollution! Quand je suis rentrée de vacances, je m'étais déshabituée, j'avais l'impression d'en avoir partout. On est comme embuées. Un nuage ... »

$\mathrm{L}:$ « La pollution : de mon appart, je suis tout en haut, je la vois. On voit un nuage noir. » T: « Un dernier point négatif sur« São Paulo c'est que l'air est très pollué, on le sent olfactivement et aussi niveau physique. Quand on bouge un peu ou qu'on veut faire un peu de sport, on sent que c'est super sec. Même quand tu marches, c'est pas bon quoi ... » M: « Le calme me manque, la circulation c'est fatiguant. Pour couper je vais dans ma chambre, je mets mes écouteurs. "

Outre ces nuisances, le règne de l'automobile rend difficiles les déambulations, également complexifiées par l'état de la voirie- trottoirs en particulier- et par de fortes dénivellations, ce que les entretiens mettent en évidence. Hormis donc le Parc Iberapuera et la Paulista, reconnus comme les seuls endroits de la ville où l' " on peut marcher ", la ville de São Paulo apparaît comme l'antithèse du walkable urbanism, qui valorise les mobilités douces.

L: « Il y a beaucoup de problèmes qui sont liés à la corruption et aux budgets. Aussi au Brésil il y a une loi qui dit que les trottoirs sont privés donc quelquefois ça donne un aspect folklorique et sympathique dans la ville ; toutes les cinq minutes ça change. Parfois c'est catastrophique, les gens sont libres de faire ce qu'ils veulent, $y^{\prime}$ a vraiment pas de norme ou alors c'est pas respecté. Les condominios s'ils veulent un immense arbre sur le trottoir alors ils vont mettre un arbre immense sur le trottoir. Pour un tout petit arbre, ils 
peuvent aussi mettre une énorme jardinière qui prendra la moitié du trottoir. Y'a aucune norme là-dessus. C'est quelque chose d'architecturalement catastrophique et déjà pour une personne normale c'est compliqué alors j'imagine pou une personne handicapée, c'est l'enfer parce que São Paulo c'est une ville qui monte et qui descend et comme les trottoirs privés ne sont pas entretenus, ils sont cassés et il y a des racines. »

$\mathrm{H}:$ « Par exemple les trottoirs il y en a qui sont inclinés, d'autres pas inclinés, y'en a qui sont entretenus, d'autres non, ça dépend. C'est un peu compliqué mais à part ça j'aime bien. »

$\mathrm{J}$ : « La plupart du temps on veut se balader sur les trottoirs et on finit par marcher sur la route en fait parce que les trottoirs sont vraiment trop étroits ou alors ils sont vraiment trop éclatés.»

\section{Conclusion : du « non lieu » à l' « hyper urbain »}

41 São Paulo est donc "l'urbain ", décrite tantôt par des repères affectifs car espace de vie, de sorties, tantôt par des maux urbains, ressentis par les lycéens. Par les critères de description mis à jour plus haut, il est intéressant de constater que la ville de São Paulo telle qu'elle est dessinée et décrite par les adolescents interrogés peut souvent apparaître comme un "non lieu», c'est-à-dire ici que l'imprécision géographique des éléments dessinés ne permet pas d'en faire un lieu «situé ». En d'autres termes que ceux de Melvin M. Webber on pourrait ici parler de «l'érosion du localisme et la déspatialisation de la ville» (Webber, 1998), d'un « urbain sans lieu, ni bornes », ce que l'absence de localisation, comme le sentiment d'une mer de béton avec un horizon borné par les immeubles peut suggérer. São Paulo est à ce titre la ville telle que la définit aussi Olivier Mongin, «ville mouvante-pieuvre» (Mongin, 2013), celle qu'il définit par trois adjectifs: anarchique, atopique et a-centrée. D'autre part, ce même auteur définit ce type de métropole comme «hyper-lieu» du fait de la sur-territorialisation des flux et les caractéristiques de l'émergence: verticalité, recherche de la hauteur et de la connexion maximale. Nous pourrions aussi parler pour São Paulo d'« hyper urbain » par la récurrence des éléments mis à jour : jungle de béton, verticalisation, pollution, ségrégation.

De la ville atopique à la sursémantisation de l'espace, on assiste dans les représentations de ces adolescents à un tiraillement dialectique entre ces deux extrêmes. A ce titre, on se rend bien compte de l'influence des pratiques spatiales sur les représentations de l'espace et du passage de l'espace pratiqué à l'espace idéel.

\section{BIBLIOGRAPHIE}

DE SOUZA M. A. A. São Paulo: ville mondiale et urbanisme français sous les tropiques, L'Harmattan, 1998.

DI MEO G., 1991. L'Homme, la Société, l'Espace. Paris, Anthropos, 319 p.

FREMONT A., 1999. La région, espace vécu. Paris, Flammarion, 288 p. 
GOULD P., WHITE R., 1974. Mental Maps. New York, Penguin Books, 187 p.

GOULD P., WHITE R., 1984. Cartes Mentales, traduit de l'anglais par A. Perroud et M. Roten.

Fribourg, Editions Universitaires de Fribourg Suisse, 169 p.

LEMAHN-FRISCH S., AUTHIER J-Y, DUFAUX F., « Draw me your neighbourhood: a gentrified Paris neighbourghood through its children's eyes", in Children's Geographies, 10:1, 17-34, 2012.

hal-univ-paris10.archives-ouvertes.fr.

LEVY J., L'invention du monde. Une géographie de la mondialisation, Paris, Presses de Sciences Po (sous la dir.), 2008.

LYNCH K. (1960) The Image of the City Cambridge, MIT Press, $194 \mathrm{p}$.

MONGIN O., La ville des flux. L'envers et l'endroit de la mondialisation urbaine, Fayard, 2013.

RIGOLLET N.A., Adolescências em Sampa, Représentations, mobilités et territoires des adolescents expatriés à São Paulo, Mémoire de recherche de deuxième année, Master II Pays en voie de développement et émergents, Université Paris I Sorbonne, soutenu le 11 septembre 2015.

SANTOS, M., Metropole corporativa fragmentada : o caso de São Paulo, São Paulo, Secretaria de Estado da Cultura, 117 p, 1990.

SCHWARTZ, O., L'empirisme irréductible. Post-face du Hobo : sociologie du sans-abri, N.Anderson, Paris, Nathan, 1993.

STASZAK J-F., 2003. Carte mentale. In Lévy J., Lussault M. (dir.), Dictionnaire de la géographie et de l'espace des sociétés. Paris, Belin, p. 132-133.

WEBBER W. M., L'urbain sans lieu ni bornes, éditions de l'Aube, Paris, 1998.

\section{NOTES}

1. Paris 1, La Sorbonne.

2. Le nombre d'inscrits au registre des Français tenu par les consulats au 31 décembre 2016 s'élève à 1782 188, soit une progression de 4,16 \% par rapport à 2015. Le taux de croissance était de 1,8\% entre 2014 et 2015 (source : data.gouv.fr).

3. «Sampa » est le surnom fréquemment donné à São Paulo par ses habitants.

\section{RÉSUMÉS}

A partir de cartes mentales et d'entretiens issus d'une enquête de terrain menée au lycée français de São Paulo en août 2014, ce travail de terrain questionne les perceptions et représentations de la ville d'adolescents français en situation d'expatriation au Brésil. Cet article souligne la construction d'une territorialité affective et sensorielle et la perception d'un espace symbolique s'affranchissant de la logique situationnelle.

From mental maps and interviews based upon a field survey conducted at Sao Paulo's French International School in August 2014, this paper deals with the perception and representation of the city through the eyes of expatriate teenagers. This paper stresses and underlines the 
construction of an emotional and sensory territoriality and the perception of a symbolic space freed from any situational logic.

INDEX

Thèmes : Carnets de terrain

Keywords : Spatial representations, Teenagers, Mental maps, Expatriation

Mots-clés : représentations spatiales, adolescents, carte mentale, expatriation

\section{AUTEUR}

NOLWENN AZILIS RIGOLLET

Université Paris 1, Laboratoire Géographie-cités

Géographie

Nolwenn-Azilis.Rigollet@univ-paris1.fr 